\title{
Contrast enhanced ultrasonography (CEUS) a novel tool to detect intestinal epithelial barrier dysfunction in severe COVID-19 disease
}

\author{
H.C. Tews ${ }^{\mathrm{a}}$, A. Kandulski ${ }^{\mathrm{a}}$, S. Schmid ${ }^{\mathrm{a}}$, G. Peschel ${ }^{\mathrm{c}}$, K. Gülow ${ }^{\mathrm{a}}$, S. Schlosser, S. Schirner ${ }^{\mathrm{a}}$, \\ C. Stroszczynski ${ }^{\mathrm{b}}$, M. Müller ${ }^{\mathrm{a}}$ and E.M. Jung \\ a Department of Internal Medicine I, Gastroenterology, Hepatology, Endocrinology, Rheumatology and \\ Infectious diseases, University Hospital, Regensburg, Germany \\ ${ }^{\mathrm{b}}$ Institute of Diagnostic Radiology, University Hospital, Regensburg, Germany \\ c Departement of Internal Medicine, Fürstenfeldbruck, Germany
}

\begin{abstract}
Ten patients with confirmed COVID-19 disease were studied. Nine patients required intensive care treatment, among them four needed extracorporeal membrane oxygenation (ECMO). Contrast enhanced ultrasonography (CEUS) was performed by one experienced investigator as a bolus injection of up to $2.4 \mathrm{ml}$ of sulphur hexafluoride microbubbles via a central venous catheter. B-Mode evaluation and strain elastography showed mural edema of the small bowel with a thickness of up to $10 \mathrm{~mm}$ in all patients. We applied color coded Doppler sonography (CCDS) and power mode with flow-adapted parameters and early, dynamic capillary arterial contrast enhancement of bowel wall structures $<10 \mathrm{~s}$ to assess perfusion of the small bowel. In all patients, reactive hyperemia was seen in the entire small bowel. In a subgroup of seven patients microbubbles translocated into the intestinal lumen. Thus, high-grade intestinal barrier disruption secondary to SARSCoV-2 infection can be postulated in these patients.

This is the first description of perfusion changes and a disruption of the small bowel epithelial barrier in COVID-19 Patients using contrast ultrasonography and elastography.
\end{abstract}

\section{Introduction}

The COVID-19 pandemic is a challenge for our society on several levels: psychologically, physically, and economically. For healthcare professionals, the burden is particularly high. The clinical manifestation from SARSCoV-2 infection known as COVID-19 disease may be asymptomatic or mild but can lead to a severe form of COVID-19 disease with acute respiratory distress syndrome (ARDS) and lethal multi-organ involvement [1].

With growing knowledge, SARSCoV-2 infection is being conceptualized as a systemic disease $[2,3]$. The current literature suggests that the SARSCoV-2 virus can affect different organ systems and causes dysregulation of the gastrointestinal tract, liver, biliary tract and pancreas [4]. There is

\footnotetext{
*Corresponding author: Hauke Christian Tews, Department of Internal Medicine I, Gastroenterology, Hepatology, Endocrinology, Rheumatology and Infectious diseases, University Hospital, Franz-Josef-Strauß-Allee 11, 93053 Regensburg, Germany. Tel.: +49 0941944 17164; Fax: +49 0941944 7107; E-mail: Hauke.Tews@ukr.de.
} 
growing evidence that a significant number of patients exhibit abdominal symptoms [5, 6]. Adults as well as up to $50 \%$ of children display gastrointestinal (GI) symptoms, which include nausea, anorexia, vomiting, diarrhea, and abdominal pain [7]. GI symptoms might precede other symptoms or even be the major manifestation of the disease and, thus, render diagnosis challenging [8, 9]. Pathophysiological mechanisms of COVID-19 appear to involve vascular, immunological and prothrombotic factors. Induction of a cytokine storm leads to endothelial damage and thrombosis, accompanied by remodeling of the vascular tissue and resulting hypoperfusion [10-12].

In the work presented here, we investigate the impact of SARSCoV-2 infection on the small and large bowel using state-of-the-art advanced ultrasound methods.

The performance of ultrasound examinations including contrast-enhanced sonography had been determined by the attending physicians at the intensive care units (ICU) in cooperation with an interdisciplinary board. CEUS investigations of critically ill patients at the ICU and the performance of intraoperative CEUS are supported by various clinical trials and the new EFSUMB guidelines [13, 14]. All examinations were performed by one experienced investigator (who has performed more than 2500 examinations/year) using a mobile ultrasound unit (LOGIQ S9) with the possibility to store dynamic digital cine-loops in DICOM format for CEUS. The requirements for ultrasound examinations of patients with COVID-19 can only be achieved using high-performance ultrasound equipment exhibiting maximum mobility while maintaining the highest standards of hygiene and infection prevention. Cross-sectional imaging is an alternative imaging modality of the abdomen for COVID-19 patients. Disadvantages are high costs, radiation exposure and high personnel deployment $[35,36,46]$.

With regard to intestinal diagnostics CEUS is of particular importance in case of diagnosis and follow-up of Crohn's disease, ulcerative colitis and for the detection of permeability disorders in Graft versus Host disease (GVHD) [15-17]. Since CEUS can image micro vascularization down to the capillary level using modern 2nd generation ultrasound contrast agents, it is also crucial for the assessment of pathologic organ alterations during the severe course of COVID-19 infection.

\section{Material and methods}

\subsection{Patients}

We have analyzed ten patients with severe SARSCoV-2 infection displaying COVID-19 ARDS (Table 1 and 2). Nine patients were mechanically ventilated and four patients on ECMO therapy. Intestinal barrier disruption was suspected because of the clinical performance status of the patients at the ICU. A state-of-the-art ultrasound examination including strain elastography and CEUS was performed. The study was approved by the local ethics committee of the University of Regensburg, Regensburg, Germany (21-2693-104).

\subsection{Ultrasound Examination}

Ultrasonography of the abdomen was performed according to the protocol previously described [18]. In summary, a sweep B-mode scan was applied using a multifrequency convex sector transducer (1-6 MHz, LOGIQ E9, GE, Milwaukee, WI, USA or LOGIQ S8, GE, Milwaukee, WI, USA) to detect potentially affected areas of the small and large bowel.

The bowel was initially examined for segmental or generalized thickening of the bowel wall $>3 \mathrm{~mm}$ with associated mural edema with or without accompanying free fluid, concomitant lymphadenopathy, hyperemia, and enlargement of intestinal fat. Color-coded Doppler sonography (CCDS) and power Doppler were used to screen for hypervascularized areas. 


\section{Table 1}

Demographic, clinical data and the outcome of the patients included in the study

\begin{tabular}{|c|c|}
\hline Demographics and Characteristics of the Patients & Patients $(n=10)$ \\
\hline Median age, y & 54,5 \\
\hline Mean age, y (SD) & $53,6(13,8)$ \\
\hline \multicolumn{2}{|l|}{ Sex } \\
\hline Male $(\%)$ & $7(70)$ \\
\hline Female $(\%)$ & $3(30)$ \\
\hline \multicolumn{2}{|l|}{ Weight $[\mathrm{BMI}](\%)$} \\
\hline Normal weight $[18,5-24,9]$ & $3(30)$ \\
\hline Overweight $[25-29,9]$ & $3(30)$ \\
\hline Obese class I [30 - 34,9] & $2(20)$ \\
\hline Obese class II $[35-39,9]$ & $0(0)$ \\
\hline Obese class III $[\geq 40]$ & $2(20)$ \\
\hline Median body mass index $\mathrm{kg} / \mathrm{m}^{2}$ & 29,2 \\
\hline Mean body mass index $\mathrm{kg} / \mathrm{m}^{2}(\mathrm{SD})$ & $30,2(7,0)$ \\
\hline \multicolumn{2}{|l|}{ Concomitant disease } \\
\hline Hypertension & $3(30)$ \\
\hline Diabetes mellitus & $2(20)$ \\
\hline Chronic kidney disease & $3(30)$ \\
\hline Chronic heart failure & $1(10)$ \\
\hline Oncologic underlying disease & $1(10)$ \\
\hline \multicolumn{2}{|l|}{ Vaccination status $(\%)$} \\
\hline Unvaccinated & $8(80)$ \\
\hline Simply vaccinated & $0(0)$ \\
\hline Double vaccinated & $2(20)$ \\
\hline Boosted & 0 \\
\hline Median days since first detection of infection with SARS-CoV-2 & 38,5 \\
\hline Mean days since first detection of infection with SARS-CoV-2 (SD) & $37,2(13,9)$ \\
\hline \multicolumn{2}{|l|}{ Performance of COVID-Sequencing (\%) } \\
\hline Yes & $4(40)$ \\
\hline No & $6(60)$ \\
\hline \multicolumn{2}{|l|}{ Virus-variant $(\%)$} \\
\hline Delta & $4(40)$ \\
\hline Omikron & $0(0)$ \\
\hline Unknown & $6(60)$ \\
\hline \multicolumn{2}{|l|}{ Non-invasive Ventilation (NIV) at the time of examination (\%) } \\
\hline Yes & $0(0)$ \\
\hline No & $10(100)$ \\
\hline Median duration NIV before, $\mathrm{d}$ & 3 \\
\hline \multicolumn{2}{|l|}{ Ventilator dependent at the time of examination (\%) } \\
\hline Yes & $9(90)$ \\
\hline No & $1(10)$ \\
\hline Median time on the ventilator, $\mathrm{d}$ & 29,5 \\
\hline \multicolumn{2}{|l|}{ ECMO at the time of examination (\%) } \\
\hline Yes & $4(40)$ \\
\hline No & $6(60)$ \\
\hline Median time on ECMO, $d$ & 18,5 \\
\hline \multicolumn{2}{|l|}{ Dialysis } \\
\hline Yes & $5(50)$ \\
\hline No & $5(50)$ \\
\hline Median time since start of dialysis, $d$ & 0,5 \\
\hline
\end{tabular}

BMI, body mass index; SD, standard deviation. 
Table 2

B-Mode/bowel wall thickness: 0 (3-4 mm, normal), 1 (5-6 mm, little), 2 (7-8 mm, moderate), 3 (>8 mm, severe); Elastography: 0 (normal), 1 (indurated), 2 (widened with edema), 3 (homogenous soft); CEUS: contrast-enhanced ultrasound: y (yes), n (no); Invasive device medicine at the time of diagnosis: D (Dialysis) E (ECMO) (I) Intubated and ventilated (S) Spontaneous breathing without circulatory support (S)

\begin{tabular}{|c|c|c|c|c|c|c|c|c|c|c|}
\hline Patient & Age, y & $\begin{array}{l}\text { Sex (female/ } \\
\text { male) }\end{array}$ & $\begin{array}{l}\text { Days since } \\
\text { infection with } \\
\text { SARS-CoV2 } \\
\text { at the time of } \\
\text { US }\end{array}$ & B-Mode & CEUS & Elastography & $\begin{array}{l}\text { Invasive } \\
\text { device } \\
\text { medicine at } \\
\text { the time of } \\
\text { US }\end{array}$ & $\begin{array}{l}\text { SARS-CoV- } \\
\text { 2-RNA from } \\
\text { respiratory } \\
\text { material at the } \\
\text { time of US, } \\
\text { cop./ml }\end{array}$ & $\begin{array}{l}\text { SARS-CoV- } \\
\text { 2-RNA in } \\
\text { Serum } \\
\text { samples at the } \\
\text { time of US, } \\
\text { cop./ml }\end{array}$ & Outcome \\
\hline 1 & 45 & $\mathrm{f}$ & 35 & 3 & $\mathrm{y}$ & 2 & $\mathrm{I}, \mathrm{E}$ & 0 & 0 & Deceased \\
\hline 2 & 35 & $\mathrm{f}$ & 39 & 2 & $\mathrm{y}$ & 2 & I & $1,2 \times 10^{3}$ & 0 & ICU-Treatment \\
\hline 3 & 81 & $\mathrm{f}$ & 8 & 2 & $\mathrm{y}$ & 2 & $\mathrm{~S}$ & $2,9 \times 10^{6}$ & - & Hospital discharge \\
\hline 4 & 62 & $\mathrm{~m}$ & 44 & 2 & $\mathrm{y}$ & 2 & I, E, D & 0 & 0 & ICU-Treatment \\
\hline 5 & 30 & $\mathrm{~m}$ & 34 & 2 & $\mathrm{y}$ & 2 & I, E, D & 0 & 0 & ICU-Treatment \\
\hline 6 & 54 & $\mathrm{~m}$ & 46 & 2 & $\mathrm{y}$ & 2 & $\mathrm{I}, \mathrm{E}$ & 0 & 0 & ICU-Treatment \\
\hline 7 & 53 & $\mathrm{~m}$ & 38 & 1 & $\mathrm{y}$ & 1 & I & 0 & 0 & $\begin{array}{l}\text { Transfer to other } \\
\text { hospital for Weaning }\end{array}$ \\
\hline 8 & 55 & $\mathrm{~m}$ & 39 & 1 & $\mathrm{y}$ & 1 & I & $10^{3}$ bis $10^{6}$ & 0 & $\begin{array}{l}\text { Transfer to ICU in } \\
\text { referring hospital }\end{array}$ \\
\hline 9 & 60 & $\mathrm{~m}$ & 65 & 2 & $\mathrm{y}$ & 1 & $\mathrm{I}, \mathrm{D}$ & 0 & 0 & ICU-Treatment \\
\hline 10 & 61 & $\mathrm{~m}$ & 24 & 2 & $\mathrm{y}$ & 2 & I & 0 & 0 & ICU-Treatment \\
\hline
\end{tabular}


Intestinal loops that showed wall thickening $>3 \mathrm{~mm}$ and edema were then examined with a highresolution linear multifrequency transducer (6-9 MHz) LOGIQ E9, GE, Milwaukee, WI, USA) to clearly distinguish intestinal loops with acute edema from those with chronic changes of the intestinal wall. In addition, we performed color-coded strain elastography.

\subsection{Strain Elastography}

Color-coded strain elastography was performed in bowel areas suspicious for bowel wall barrier disruption based on B-mode findings, as previously described [18]. To exclude fibrous stenoses, strain elastography was additionally performed in acute reactive bowel wall lesions to show soft bowel wall portions.

As such, elastography implemented in the high-end ultrasound device allows assessment and visualization of elastic properties and stiffness of tissue acting against shear deformation.

While examining the intestinal area of interest with B-mode ultrasound, a strain elastogram is generated by applying compression and decompression and by visualizing the resulting changes in the B-mode image. The image is transferred into a color-coded map using a scale from red (high strain, soft) to blue (low strain, hard).

As a result, scar formation appears with higher stiffness coded in blue as opposed to acute intestinal inflammatory regions displayed by tissue softening coded in red or yellow [19, 20].

\subsection{CEUS}

Contrast-enhanced ultrasound (CEUS) was applied by intravenous bolus injection of 1.5-2.4 mL Sulphur hexafluoride microbubbles (SonoVue, BRACCO, Italy) via a peripheral venous catheter followed by a $10 \mathrm{~mL}$ saline solution flush. DICOM loops up to $1 \mathrm{~min}$ were continuously stored, starting from the early arterial phase after $10-15 \mathrm{~s}$ up to $1 \mathrm{~min}$ and then short loops up to $10 \mathrm{~s}$ until the late phase (up to $5 \mathrm{~min}$ ) and were digitally stored as DICOM loops of approximately $10 \mathrm{~s}$ for independent retrospective reading by two experienced ultrasound examiners.

Incipient contrast of the mesenteric tissue and bowel wall was considered the reference. Rapid contrast $<10$ seconds was regarded as hyperemia with also increased flow signal in the duplex. For this purpose, flow parameters were optimized for low venous flow components, scale $<1000 \mathrm{~Hz}$, wall filter $<100$, optimization of the gain to the onset of artifacts or aliasing.

Evaluation of the intestinal loops regarding the contrast agent behavior was performed as previously described by our group [15]. Briefly, by implementing pulse inversion harmonic imaging (PIHI) and reduced mechanical index $(\mathrm{MI}<0.2)$ to exclude artefacts, translocation of microbubbles into the intestinal lumen was interpreted as a consequence of a SARSCoV-2 manifestation.

\subsection{Ultrasound imaging analysis}

B-mode ultrasound, strain elastography and CEUS was performed and interpreted during the same investigation by an experienced examiner. Whether microbubble penetration into the bowel lumen as a correlate of a significant intestinal barrier disorder was observed, was evaluated independently by two experienced examiners. Observed abnormalities in bowel wall thickness on B-mode (coding 0 (3-4 mm), 1 (5-6 mm, little), 2 (7-8 mm, moderate) $(3>8 \mathrm{~mm}$, severe) and strain elastography were coded from 0 to 3 to assess severity (coding: 0 normal), 1 (indurated), 2 (widened with edema), 3 (homogenous soft). 
I

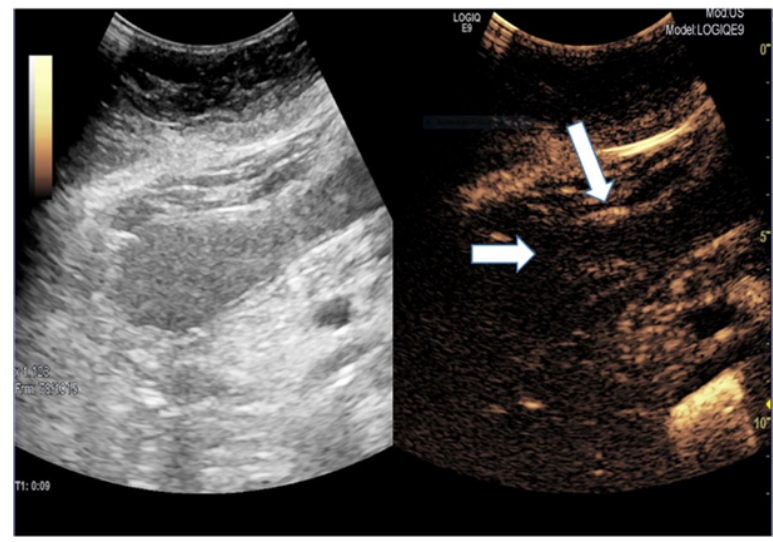

II

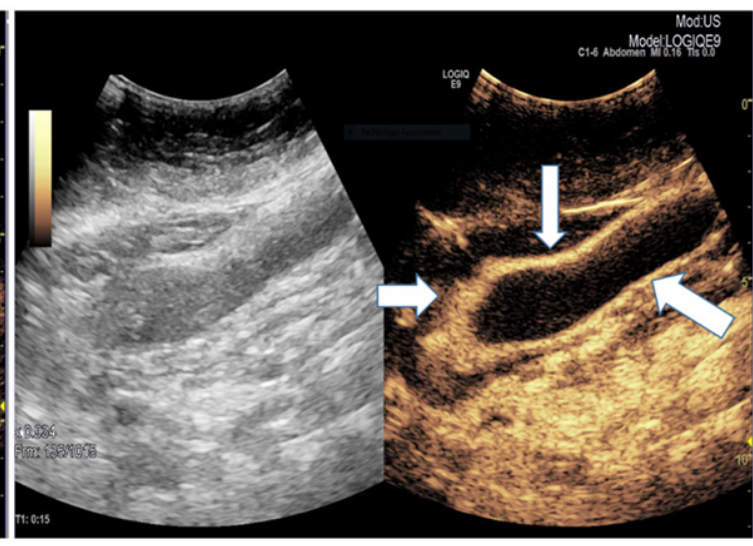

Fig. 1. Ultrasound images of a 45-year-old patient with severe COVID-19 disease. I: B Mode ultrasound showing intestinal wall thickening; CEUS image showing mild arterial enhancement 9 seconds after SonoVue application (white arrows). II: CEUS image showing early and strong arterial hyperenhancement of the intestinal wall (white arrow) 15 seconds after SonoVue application.

\section{Results}

We analyzed ten patients with severe COVID-19 infection (Table 1/2). Ultrasound-based diagnosis of significant intestinal barrier disorder was established by two experienced ultrasound examiners based on the combined evaluation of intestinal wall thickening in B-mode ultrasound, hypervascularization in color-coded doppler sonography and early arterial enhancement and transmural translocation of microbubbles in CEUS as described before [18].

We observed morphological signs of pathological bowel wall morphology in all patients. Signs such as hypervascularization, bowel wall edema, contrast enhancement and even intraluminal microbubble accumulation were present with varying degrees (Table 2). Interestingly, Figs. 1, 2, 4, and 5 show ubiquitous, severe, early arterial hyperenhancement. This is a sign of hypervascularization, probably because of the systemic inflammatory response.

In Fig. 1, a CEUS image of a 45-year-old female patient is presented. The patient suffered from severe COVID-19 ARDS without relevant medical history and was treated by VV-ECMO. The images demonstrate an early, almost fulminant accumulation of contrast agent in the wall of the small intestine 15 seconds after contrast application.

Figure 2 shows Ultrasound and CT images of the same patient (Fig. 1). 21 seconds after contrast agent administration, a strong contrast accumulation of the intestinal wall can still be observed by CEUS. Of note, in Figure, II isolated microbubbles can be detected in distinct areas of the intestinal wall. CT images confirm small bowel pathology with thickening of the small bowel wall, associated edema and hypervascularization (Fig. 2).

Figure 3 shows the different contrast uptake of the intestinal wall layers in a 45-year-old patient severely affected by COVID-19 at 5, 10, and 14 seconds after SonoVue application. At 5 seconds after SonoVue application, intersegmental transmural contrast uptake was noted. At 10 seconds and 14 seconds after SonoVue application, a complete, long-range contrast image of the intestinal wall layers is shown.

In Fig. 4, elastography, CEUS and CT images of a 30-year-old patient severely affected by COVID-19 are shown, respectively. In these images we observe again an early arterial contrast hyperenhancement in the intestinal wall, in elastography a soft wall edema and a translocation of microbubbles into the 
I

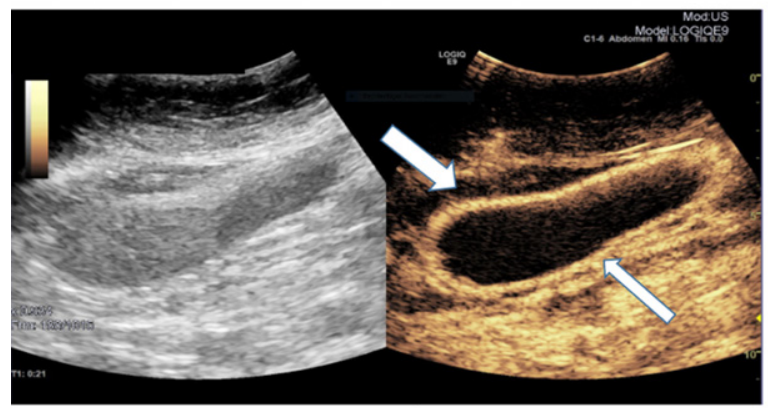

II

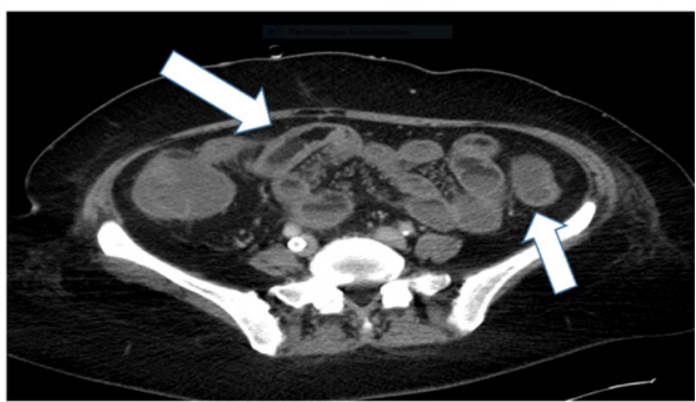

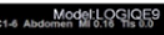

III

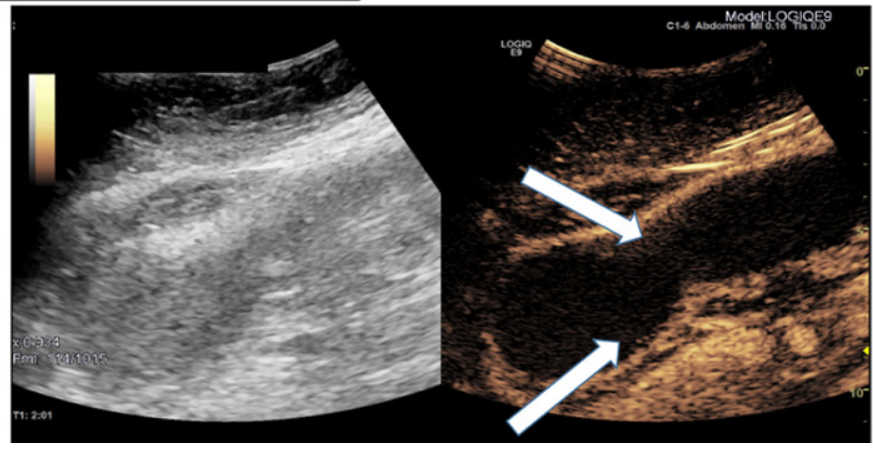

Fig. 2. Ultrasound images of a 45-year-old patient with severe COVID-19 disease. I: B-Mode ultrasound showing intestinal wall thickening. CEUS image manifesting early and strong arterial hyperenhancement of the intestinal wall (white arrows) 21 seconds after SonoVue application. II: Computertomography (CT) image depicting bowel wall edema and contrast enhancement of the bowel wall (white arrows). III: CEUS imaging expressing transmural penetration of microbubbles (white arrows).

lumen. The CT of the lung, 39 days after initial COVID-19 detection, illustrates the severe and structural change in the respiratory tract. The patient had no severe previous diseases before her infection with SARSCoV-2.

Figure 5 shows the images of an 81-year-old female patient. The images were documented four days after the initial COVID-19 diagnosis. Interestingly, this case differs clinically from the other patients due to the milder COVID-19 course. Nevertheless, we observed a clear early arterial hyperenhancement of the intestinal wall and a crossing of microbubbles into the lumen. Elastography reveals soft edematous tissue.

Table 1 presents the demographic and clinical data of the patients included in the study. The study included ten patients with a median age of 54 years. 7 male and 3 female patients were included in the study. Seven patients suffered from obesity at different levels. $80 \%$ of the severely ill patients were not vaccinated for SARSCoV-2. The delta variant of COVID-19 represents the dominant variant in the study cohort. Four patients required ECMO therapy. Arterial hypertension $(n=3)$, diabetes mellitus $(n=2)$, and chronic kidney disease $(n=3)$ were the most common comorbidities. At the time of ultrasonography, the median diagnosis of SARSCoV-2 infection had been established 38 days prior.

Table 2 presents the results using different morphological contrast criteria.

In $n=8$ patients, elastography demonstrated bowel wall edema with a high rate of soft fractions. The contrast agent SonoVue was applied in all included patients. In $n=7$ patients, the microbubbles passed into the intestinal lumen. In $n=7$ patients, the small bowel wall was moderately widened (7-8 $\mathrm{mm}) \mathrm{on}$ native ultrasonography. In one patient even segmental thickening of the bowel wall to $10 \mathrm{~mm}$ could be observed. In addition, duration of COVID-19 disease and infectivity is shown. SARSCoV-2 RNA 
I

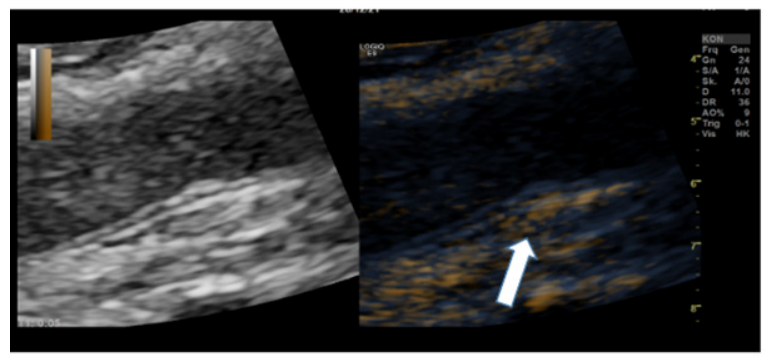

III

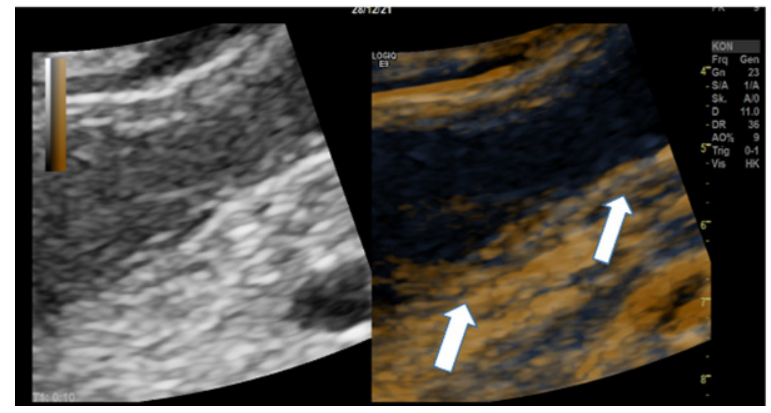

II

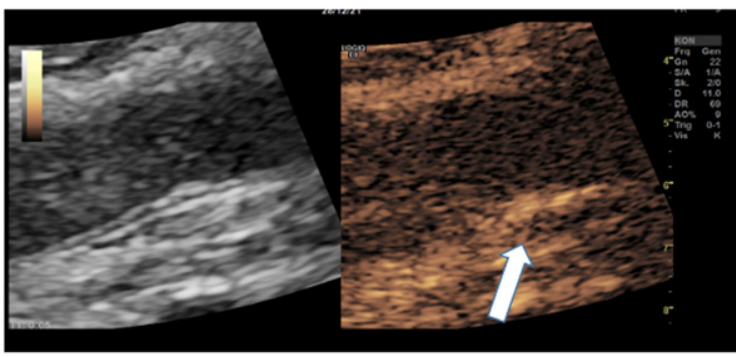

IV

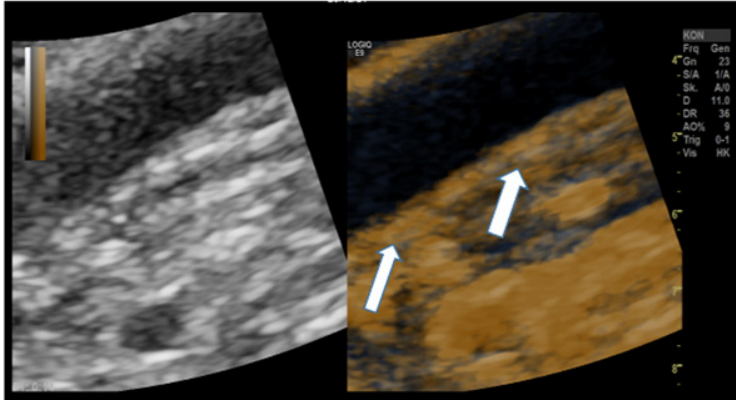

Fig. 3. Ultrasound images of a 45-years-old patient with a severe COVID-19 Infection. I: CEUS image showing early, pronounced and especially segmental, transmural arterial hyperenhancement of the intestinal wall (white arrows) 5 seconds after SonoVue application. II: CEUS image showing early, strong, and especially segmental, transmural arterial hyperenhancement of the intestinal wall (white arrows) 5 seconds after SonoVue application. III: CEUS Image shows long-distance, marked contrast uptake across all intestinal wall layers 10 seconds after SonoVue application. IV: CEUS Image shows long-distance, distinct contrast uptake across all intestinal wall layers 14 seconds after SonoVue application.

from respiratory samples and SARSCoV-2 RNA from serum samples at the time of the ultrasound examination are presented.

In conclusion, we identified pathologies of the small bowel in all included patients with severe COVID-19. These pathologies could be identified by native ultrasound, elastography and CEUS (Table 2). In particular, the high proportion of soft bowel wall edema $(n=8)$ and the detection of microbubbles in the intestinal lumen $(n=7)$ support the hypothesis of small bowel involvement in the context of COVID-19 systemic disease.

\section{Discussion}

The aim of our work was to assess the increasing evidence of GI tract involvement during a COVID-19 infection [21]. For this purpose, we applied advanced ultrasound techniques.

According to our experience, CEUS is an excellent diagnostic tool for monitoring severe cases of intermediate and intensive care patients with early changes of abdominal microcirculatory disorders and small as well as large bowel pathologies [13, 22, 23]. The advantage of CEUS with sulphur hexafluoride microbubbles is the dynamic assessment including the capillary level and strictly intravascular from the early arterial phase ( 10 to $15 \mathrm{~s}$ ) to the late phase of $5 \mathrm{~min}$. In addition, Sonovue has the decisive advantage of not having any effect on thyroid function and not being nephrotoxic [14].

In our cohort, we observed early intersegmental, transmural contrast uptake of the small bowel (Fig. 3). Subsequently, in most cases, a long segmental, band-like contrast uptake involving all intestinal wall layers of the small bowel was demonstrated after approximately 10 seconds (Figs 1/2/3/4/5). We 
I

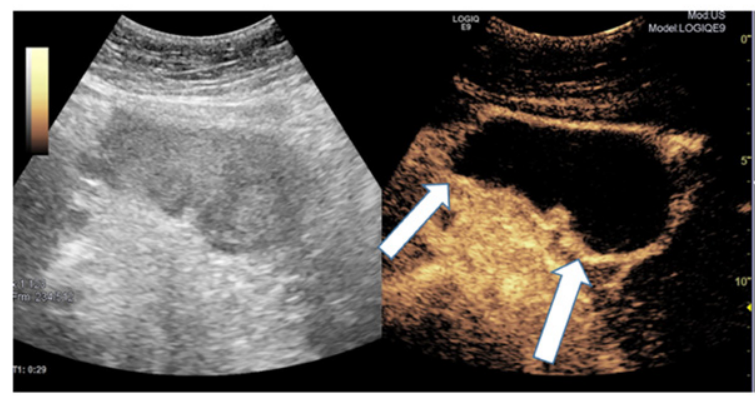

III

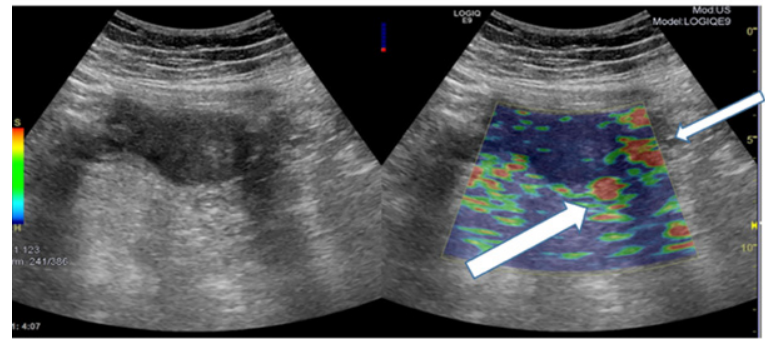

II

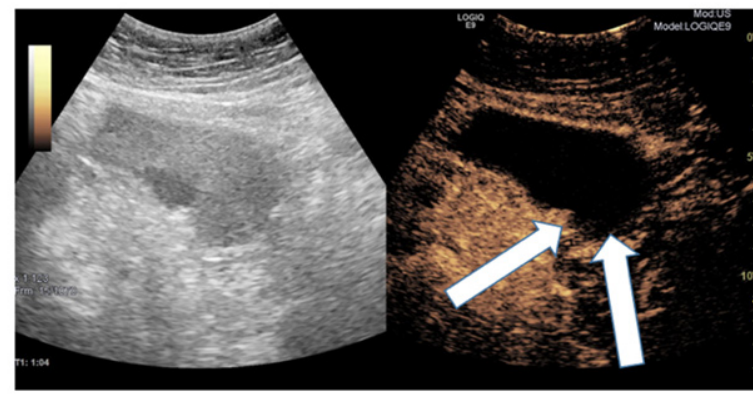

IV

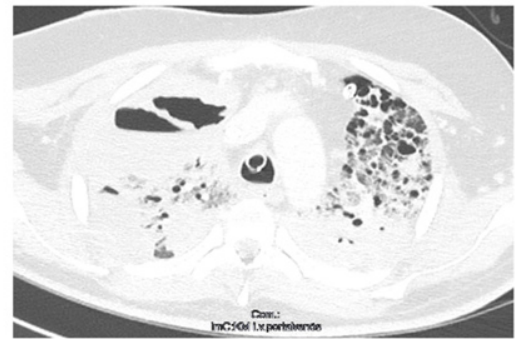

Fig. 4. Ultrasound images of a 30-years old-patient with a severe COVID-19 Infection. I: B-Mode ultrasound showing intestinal wall thickening. CEUS image showing early and strong arterial hyperenhancement of the intestinal wall (white arrows) 29 seconds after SonoVue application. II: CEUS imaging showing transmural penetration of microbubbles (white arrows). III: Soft tissue edema as a correlate of acute inflammation by strain elastography (white arrows). IV: CT of the lung. 39 days after COVID-19 diagnosis.

hypothesize that especially early transmural enhancement and late ubiquitous enhancement in the intestinal wall are different from other intestinal diseases. Early transmural contrast uptake of the intestinal wall layers and late-banded uptake of the complete intestinal wall in our cohort differs from the contrast behavior in GVHD and infectious enteritis [15, 18, 24]. It is conceivable that the early transmural involvement of the intestinal wall in the setting of COVID-19 disease has similarities to a severe episode of inflammatory bowel disease [25, 26].

Limitations of the investigations are that CEUS is not able to record all intestinal structures in their microvascularization dynamically and continuously at the same time. Experienced examiners and special equipment are required. The price for $2.4 \mathrm{ml}$ ultrasound contrast medium is $40-50$ euros. The time expenditure with the necessary hygiene measures lies with up to one hour with ways and equipment for the investigation under COVID-19 protective measures counted. The absence of final pathological results of the small intestine is another limitation of the study. Despite its known limitations, modern sonography including CEUS has proven its value in times of the COVID-19 pandemic and was able to detect microcirculatory dysfunction at an early stage [27-29]. It is necessary to monitor the microcirculation at the bedside in intensive care patients who have serious COVID-19 disease [30] There is growing evidence that the combination of endothelial dysfunction with a generalized inflammatory state and complement involvement may contribute to the overall pro-coagulative state described in COVID-19 patients leading to venular as well as to arteriolar occlusions [31].

In our work, we describe pathologies of the small bowel in the setting of COVID-19 disease. Small bowel pathologies were objectified by ultrasound and quantified by contrast-enhanced ultrasound. The use of CEUS allows bedside investigations for a variety of clinical questions relevant to clinical practice. It is possible to quickly rule out organ infarction and to dynamically record the mesenteric 
I

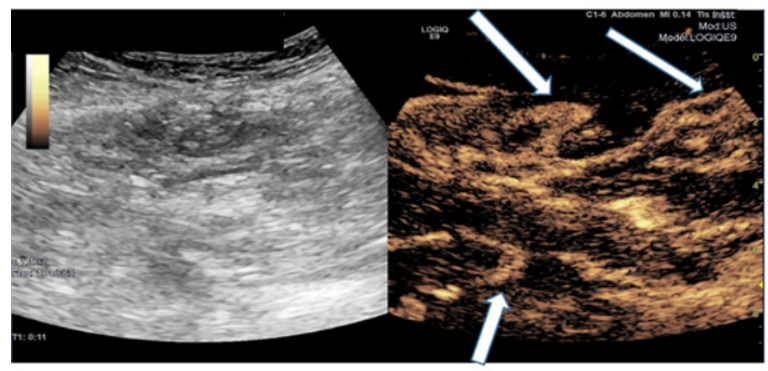

II

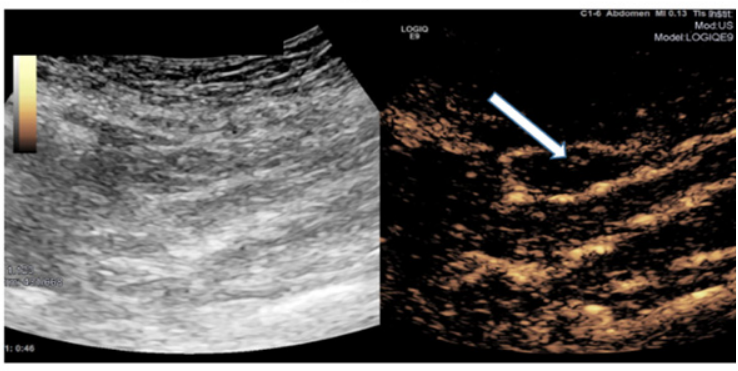

III

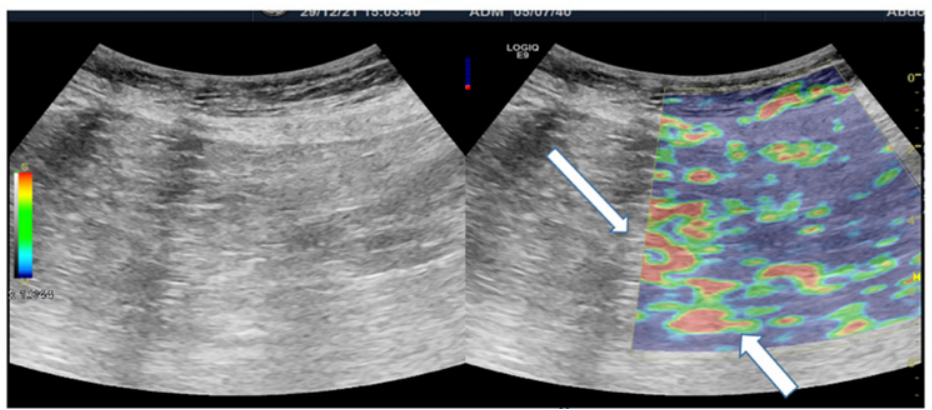

Fig. 5. Ultrasound images of a 81-years-old patient with severe COVID-19 disease. I: B-Mode ultrasound showing intestinal wall thickening. CEUS image showing early and pronounced arterial hyperenhancement of the intestinal wall (white arrows) 11 seconds after SonoVue application. II: CEUS imaging depiciting translocation of microbubbles (white arrows) into the intestinal lumen. III: Soft tissue edema as a correlate of acute inflammation diagnosed by strain elastography (white arrows).

arterial and venous blood flow [29]. CEUS can also detect peripheral reduced blood flow, microinfarctions, embolisms in the context of pulmonary artery embolism and reactive hyperemia in the case of consolidations and pleural irritation in the periphery of the lungs [32-34]. CEUS enables the dynamic assessment of organ micro- perfusion, especially of the liver, spleen and kidneys [14].

CEUS of small and large bowel has gained significant importance in recent years. CEUS of the gastrointestinal tract provides vascular information that is helpful in characterizing tumors and other pathology in and around the intestine, like contrast applications in other solid organs such as the liver. The use of microbubble contrast agents for the intestine provides additional information on mural and mesenteric blood flow, which is invaluable in determining disease activity and assessing the integrity of the intestinal barrier [16]. Small-intestine contrast enhanced Ultrasound Sonography (SICUS) has gained importance in different indications of structural bowel diseases. SICUS can be used to describe the contrast behavior of the bowel wall and to exclude possible complications such as abscesses, strictures and fistulas [17, 35].

The pathophysiology of COVID-19 is complex and the clinical picture heterogeneous. Evidence is mounting that the gut may play a central role in the acute phase of SARSCoV-2 infection and also in post-COVID-19 Syndrome [7, 36-38].

Invasive intensive care device medicine with the high number of ECMO therapies in our cohort and possible small bowel pathology secondary to perfusion deficits are potential confounders in our study but in generally not often detected in other patients with severe ARDS. There are case reports describing acute bowel ischemia during ECMO therapy. Presumably, non-occlusive or occlusive mesenteric ischemia is the cause of the bowel ischemia in these patients with ECMO therapy [39-41]. 
There are still many questions regarding the intestinal barrier function and COVID-19: Is the disruption of the integrity of the intestinal wall barrier an early feature and a major driver of systemic function or is the structural alteration of the intestinal wall barrier more likely to be an expression of severe COVID-19 infection in terms of multiorgan involvement?

In our cohort, there was no duplex sonographic hint of perfusion deficit or nonocclusive mesenteric ischemia. Thus, we conclude, that the segmental and severe transmural contrast enhancement in our studied patients is a consequence of the systemic infection in severe COVID-19 disease.

Although understanding of the gut-lung axis is in its early beginning, there is increasing evidence that altered gut microbiota and disruption of gut barrier integrity may contribute significantly to lung disease progression $[42,43]$. The high levels of circulating proinflammatory cytokines by viral infections are capable of altering gut microbiota and affecting intestinal integrity [44]. Small bowel malfunction leads to altered gut microbiota and inflammation due to the well-balanced bidirectional interaction between the gut microbiota and the immune system. Increased inflammation in the intestine leads to a leaky gut allowing bacterial antigens and toxins to translocate to the systemic circulation, further worsening the septic state of the patients with COVID-19 [45]. The mechanism of a leaky gut was described by our research group in patients with liver cirrhosis. Similarities in pathophysiology in COVID-19 patients are conceivable [55].

Dysbiosis, gut microbial metabolites and dysregulation of the intestinal barrier influence immune responses, inflammation, and disease progression of the lung [46]. The complex intercommunication through the gut-lung axis might be important in predetermining the susceptibility of the airways to SARSCoV-2 infection and COVID-19 clinical outcome as a function of the inter-individual variations in microbiota richness and composition [47].

The ACE-2 receptor is regarded to play a critical role in COVID-19 disease as a portal of entry into the lung as well as the intestinal tissue [48]. Angiotensin-converting enzyme 2 (ACE2) is not only an enzyme but also a functional receptor on cell surfaces through which SARSCoV-2 enters the host cells and is highly expressed in the heart, kidneys, lungs and the bowel and shed into the plasma. ACE-2 is a key regulator of the renin-angiotensin-aldosterone system (RAAS) [49]. The review by Zhang et el. was able to describe a distinct ACE-2 expression on small intestinal cells in COVID-19 patients [50]. Notably, the results of the study by Hamming et al. indicate that ACE-2 is present in all parts of small intestinal epithelium cells, including the duodenum, the jejunum, and the ileum, but not in enterocytes of the colon [51].

In our work, we observed an accumulation of small bowel pathologies but not of large bowel pathologies in COVID-19 patients. One explanation for this observation may be a different receptor occupancy of the ACE receptor in the small and large intestine.

Numerous records demonstrated several autoantibodies in severely ill COVID-19 patients that are normally detected in other autoimmune diseases, such as antinuclear antibodies, antiphospholipid antibodies, and many others. In addition, many case reports of COVID-19-infected patients developing autoimmune diseases have been published [52-54]. It is extremely important to consider the autoimmune effects of COVID-19 virus. It is also conceivable that autoimmune phenomena play a role in the small bowel involvement in the setting of COVID-19 disease in our cohort.

In summary, we were able to demonstrate a distinct and early contrast enhancement of the intestinal wall and the accumulation of microbubbles in the lumen of the small bowel in most patients. This finding suggests a high-grade barrier disorder of the small intestine in COVID-19 patients with a severe course. So far, our work is the first to describe an intestinal barrier disorder in patients with severe COVID-19 infection by bedside contrast ultrasonography and elastography.

In the future, it will be important to study intestinal barrier disruption and the alteration of the microbiome at different stages of the disease to better assess the role of the gut and its integrity in complex COVID-19 disease. CEUS could take a decisive position here. 


\section{Conclusion}

Intestinal barrier disorders resulting from COVID-19 are common and can be diagnosed at the bedside by multiparametric US examinations. COVID-19-specific US findings of the intestine have not been reported previously, but the reported results correlated with the clinical picture.

Our group hypothesized that intestinal wall barrier disruption is an important piece of the puzzle to understand the complex systemic disease of SARSCoV-2 infection.

\section{References}

[1] Hasöksüz M, Kiliç S, Saraç F. Coronaviruses and SARS-COV-2. Turk J Med Sci. 2020;50(SI-1):549-56.

[2] Ashraf UM, Abokor AA, Edwards JM, Waigi EW, Royfman RS, Hasan SA-M, u. a. SARS-CoV-2, ACE2 expression, and systemic organ invasion. Physiol Genomics. 2021;53(2):51-60.

[3] Synowiec A, Szczepański A, Barreto-Duran E, Lie LK, Pyrc K. Severe Acute Respiratory Syndrome Coronavirus 2 (SARS-CoV-2): a Systemic Infection. Clin Microbiol Rev. 2021;34(2):e00133-20.

[4] Hunt RH, East JE, Lanas A, Malfertheiner P, Satsangi J, Scarpignato C, u. a. COVID-19 and Gastrointestinal Disease: Implications for the Gastroenterologist. Dig Dis. 2021;39(2):119-39.

[5] Cheung KS, Hung IFN, Chan PPY, Lung KC, Tso E, Liu R, u. a. Gastrointestinal Manifestations of SARS-CoV2 Infection and Virus Load in Fecal Samples From a Hong Kong Cohort: Systematic Review and Meta-analysis. Gastroenterology. 2020;159(1):81-95.

[6] Parasa S, Desai M, Thoguluva Chandrasekar V, Patel HK, Kennedy KF, Roesch T, u. a. Prevalence of Gastrointestinal Symptoms and Fecal Viral Shedding in Patients With Coronavirus Disease 2019: A Systematic Review and Metaanalysis. JAMA Netw Open. 2020;3(6):e2011335.

[7] Mao R, Qiu Y, He J-S, Tan J-Y, Li X-H, Liang J, u. a. Manifestations and prognosis of gastrointestinal and liver involvement in patients with COVID-19: a systematic review and meta-analysis. Lancet Gastroenterol Hepatol. 2020;5(7):667-78.

[8] Pan L, Mu M, Yang P, Sun Y, Wang R, Yan J, u. a. Clinical Characteristics of COVID-19 Patients With Digestive Symptoms in Hubei, China: A Descriptive, Cross-Sectional, Multicenter Study. Am J Gastroenterol. 2020;115(5):76673.

[9] Song Y, Liu P, Shi XL, Chu YL, Zhang J, Xia J, u. a. SARS-CoV-2 induced diarrhoea as onset symptom in patient with COVID-19. Gut. Juni. 2020;69(6):1143-4.

[10] Henry BM, Vikse J, Benoit S, Favaloro EJ, Lippi G. Hyperinflammation and derangement of renin-angiotensinaldosterone system in COVID-19: A novel hypothesis for clinically suspected hypercoagulopathy and microvascular immunothrombosis. Clin Chim Acta. 2020;507:167-73.

[11] Becker RC. COVID-19-associated vasculitis and vasculopathy. J Thromb Thrombolysis. 2020;50(3):499-511.

[12] McFadyen JD, Stevens H, Peter K. The Emerging Threat of (Micro)Thrombosis in COVID-19 and Its Therapeutic Implications. Circ Res. 2020;127(4):571-87.

[13] Sidhu PS, Cantisani V, Dietrich CF, Gilja OH, Saftoiu A, Bartels E, u. a. The EFSUMB Guidelines and Recommendations for the Clinical Practice of Contrast-Enhanced Ultrasound (CEUS) in Non-Hepatic Applications: Update 2017 (Long Version). Ultraschall Med. 2018;39(2):e2-44.

[14] Dietrich CF, Averkiou M, Nielsen MB, Barr RG, Burns PN, Calliada F, u. a. How to perform Contrast-Enhanced Ultrasound (CEUS). Ultrasound Int Open. 2018;4(1):E2-15.

[15] Pausch A-M, Kammerer S, Weber F, Herr W, Stroszczynski C, Holler E, u. a. Parametric Imaging of Contrast-Enhanced Ultrasound (CEUS) for the Evaluation of Acute Gastrointestinal Graft-Versus-Host Disease. Cells. 2021;10(5):1092.

[16] Medellin A, Merrill C, Wilson SR. Role of contrast-enhanced ultrasound in evaluation of the bowel. Abdom Radiol (NY). 2018;43(4):918-33.

[17] Mocci G, Migaleddu V, Cabras F, Sirigu D, Scanu D, Virgilio G, u. a. SICUS and CEUS imaging in Crohn's disease: an update. J Ultrasound. 2017;20(1):1-9.

[18] Weber D, Weber M, Hippe K, Ghimire S, Wolff D, Hahn J, u. a. Non-invasive diagnosis of acute intestinal graft-versushost disease by a new scoring system using ultrasound morphology, compound elastography, and contrast-enhanced ultrasound. Bone Marrow Transplant. 2019;54(7):1038-48.

[19] Dietrich CF, Bamber J, Berzigotti A, Bota S, Cantisani V, Castera L, u. a. EFSUMB Guidelines and Recommendations on the Clinical Use of Liver Ultrasound Elastography, Update 2017 (Long Version). Ultraschall Med. 2017;38(4):e16-47. 
[20] Ozturk A, Grajo JR, Dhyani M, Anthony BW, Samir AE. Principles of ultrasound elastography. Abdom Radiol (NY). 2018;43(4):773-85.

[21] Tirumani SH, Rahnemai-Azar AA, Pierce JD, Parikh KD, Martin SS, Gilkeson R, u. a. Are asymptomatic gastrointestinal findings on imaging more common in COVID-19 infection? Study to determine frequency of abdominal findings of COVID-19 infection in patients with and without abdominal symptoms and in patients with chest-only CT scans. Abdom Radiol (NY). 2021;46(6):2407-14.

[22] Lerchbaumer MH, Putz FJ, Rübenthaler J, Rogasch J, Jung E-M, Clevert D-A, u. a. Contrast-enhanced ultrasound (CEUS) of cystic renal lesions in comparison to CT and MRI in a multicenter setting. Clin Hemorheol Microcirc. 2020;75(4):419-29.

[23] Lerchbaumer MH, Kleemann T, Jung E-M, Nagel S, Hamm B, Fischer T. Vascular pattern and diagnostic accuracy of contrast-enhanced ultrasound (CEUS) in spleen alterations. Clin Hemorheol Microcirc. 2020;75(2):177-88.

[24] Schreyer AG, Landfried K, Jung EM, da Silva NPB, Poschenrieder F, Dornia C, u. a. Contrast-enhanced ultrasound for differential diagnosis of suspected GvHD in patients after allogeneic transplantation. Clin Hemorheol Microcirc. 2011;49(1-4):129-36.

[25] Pecere S, Holleran G, Ainora ME, Garcovich M, Scaldaferri F, Gasbarrini A, u. a. Usefulness of contrast-enhanced ultrasound (CEUS) in Inflammatory Bowel Disease (IBD). Dig Liver Dis. 2018;50(8):761-7.

[26] Romanini L, Passamonti M, Navarria M, Lanzarotto F, Villanacci V, Grazioli L, u. a. Quantitative analysis of contrastenhanced ultrasonography of the bowel wall can predict disease activity in inflammatory bowel disease. Eur $\mathbf{J}$ Radiol. 2014;83(8):1317-23.

[27] Jung EM, Stroszczynski C, Jung F. Contrast enhanced ultrasound (CEUS) to assess pleural pulmonal changes in severe COVID-19 infection: First results. Clin Hemorheol Microcirc. 2020;75(1):19-26.

[28] Peschel G, Jung EM, Fisser C, Putz FJ, Wertheimer T, Sinner B, u. a. Interstitial lung opacities in patients with severe COVID-19 pneumonia by bedside high-resolution ultrasound in association to CO2 retention. Clin Hemorheol Microcirc. 2021;77(4):355-65.

[29] Jung EM, Stroszczynski C, Jung F. Contrast enhanced ultrasonography (CEUS) to detect abdominal microcirculatory disorders in severe cases of COVID-19 infection: First experience. Clin Hemorheol Microcirc. 2020;74(4):353-61.

[30] Martini R. The compelling arguments for the need of microvascular investigation in COVID-19 critical patients. Clin Hemorheol Microcirc. 2020;75(1):27-34.

[31] Jung F, Krüger-Genge A, Franke RP, Hufert F, Küpper J-H. COVID-19 and the endothelium. Clin Hemorheol Microcirc. 2020;75(1):7-11.

[32] Watchorn J, Huang DY, Joslin J, Bramham K, Hutchings SD. Critically Ill COVID-19 Patients With Acute Kidney Injury Have Reduced Renal Blood Flow and Perfusion Despite Preserved Cardiac Function: A Case-Control Study Using Contrast-Enhanced Ultrasound. Shock. 2021;55(4):479-87.

[33] Safai Zadeh E, Görg C, Dietrich CF, Görlach J, Alhyari A, Trenker C. Contrast-Enhanced Ultrasound for Evaluation of Pleural Effusion: A Pictorial Essay. J Ultrasound Med. 2021;

[34] Safai Zadeh E, Beutel B, Dietrich CF, Keber CU, Huber KP, Görg C, u. a. Perfusion Patterns of Peripheral Pulmonary Lesions in COVID-19 Patients Using Contrast-Enhanced Ultrasound (CEUS): A Case Series. J Ultrasound Med. 2021;40(11):2403-11.

[35] Hakim A, Alexakis C, Pilcher J, Tzias D, Mitton S, Paul T, u. a. Comparison of small intestinal contrast ultrasound with magnetic resonance enterography in pediatric Crohn's disease. JGH Open. 2020;4(2):126-31.

[36] Mönkemüller K, Fry L, Rickes S. COVID-19, coronavirus, SARS-CoV-2 and the small bowel. Rev Esp Enferm Dig. 2020;112(5):383-8.

[37] Lamers MM, Beumer J, van der Vaart J, Knoops K, Puschhof J, Breugem TI, u. a. SARS-CoV-2 productively infects human gut enterocytes. Science. 2020;369(6499):50-4.

[38] Hilpert K, Mikut R. Is There a Connection Between Gut Microbiome Dysbiosis Occurring in COVID-19 Patients and Post-COVID-19 Symptoms? Front Microbiol. 2021;12:732838.

[39] Tramm R, Ilic D, Davies AR, Pellegrino VA, Romero L, Hodgson C. Extracorporeal membrane oxygenation for critically ill adults. Cochrane Database Syst Rev. 2015;1:CD010381.

[40] Huerter M, Govostis D, Ellenby M, Smith-Singares E. Acute Bowel Ischemia Associated with Left Ventricular Thrombus and Arteriovenous Extracorporeal Membrane Oxygenation. J Extra Corpor Technol. 2018;50(1):58-60.

[41] Taieb A, Jeune F, Lebbah S, Schmidt M, Deransy R, Vaillant J-C, u. a. Emergency Abdominal Surgery Outcomes of Critically Ill Patients on Extracorporeal Membrane Oxygenation: A Case-Matched Study with a Propensity Score Analysis. World J Surg. 2019;43(6):1474-82.

[42] Budden KF, Gellatly SL, Wood DLA, Cooper MA, Morrison M, Hugenholtz P, u. a. Emerging pathogenic links between microbiota and the gut-lung axis. Nat Rev Microbiol. 2017;15(1):55-63.

[43] Dang AT, Marsland BJ. Microbes, metabolites, and the gut-lung axis. Mucosal Immunol. 2019;12(4):843-50. 
[44] Hussain I, Cher GLY, Abid MA, Abid MB. Role of Gut Microbiome in COVID-19: An Insight Into Pathogenesis and Therapeutic Potential. Front Immunol. 2021;12:765965.

[45] Wang H, Ma S. The cytokine storm and factors determining the sequence and severity of organ dysfunction in multiple organ dysfunction syndrome. Am J Emerg Med. 2008;26(6):711-5.

[46] Zhang D, Li S, Wang N, Tan H-Y, Zhang Z, Feng Y. The Cross-Talk Between Gut Microbiota and Lungs in Common Lung Diseases. Front Microbiol. 2020;11:301.

[47] Donati Zeppa S, Agostini D, Piccoli G, Stocchi V, Sestili P. Gut Microbiota Status in COVID-19: An Unrecognized Player? Front Cell Infect Microbiol. 2020;10:576551.

[48] Harrison AG, Lin T, Wang P. Mechanisms of SARS-CoV-2 Transmission and Pathogenesis. Trends Immunol. 2020;41(12):1100-15.

[49] Beyerstedt S, Casaro EB, Rangel ÉB. COVID-19: angiotensin-converting enzyme 2 (ACE2) expression and tissue susceptibility to SARS-CoV-2 infection. Eur J Clin Microbiol Infect Dis. 2021;40(5):905-19.

[50] Zhang H, Li H-B, Lyu J-R, Lei X-M, Li W, Wu G, u. a. Specific ACE2 expression in small intestinal enterocytes may cause gastrointestinal symptoms and injury after 2019-nCoV infection. Int J Infect Dis. 2020;96:19-24.

[51] Hamming I, Timens W, Bulthuis MLC, Lely AT, Navis GJ, van Goor H. Tissue distribution of ACE2 protein, the functional receptor for SARS coronavirus. A first step in understanding SARS pathogenesis. J Pathol. 2004;203(2):6317.

[52] Saad MA, Alfishawy M, Nassar M, Mohamed M, Esene IN, Elbendary A. COVID-19 and Autoimmune Diseases: A Systematic Review of Reported Cases. Curr Rheumatol Rev. 2021;17(2):193-204.

[53] Dotan A, Shoenfeld Y. [COVID-19 AND AUTOIMMUNE DISEASES]. Harefuah. 2021;160(2):62-7.

[54] Galeotti C, Bayry J. Autoimmune and inflammatory diseases following COVID-19. Nat Rev Rheumatol. 2020;16(8):413-4.

[55] Haderer M, Neubert P, Rinner E, Scholtis A, Broncy L, Gschwendtner H, u. a. Novel pathomechanism for spontaneous bacterial peritonitis: disruption of cell junctions by cellular and bacterial proteases. Gut. 2021;gutjnl-2020-321663. 